\title{
Vertebral Artery Dissection as a Cause of Cervical Radiculopathy
}

\author{
Benjamin Isaac Silbert ${ }^{1}$, Mark Khangure ${ }^{2}$, Peter Linton Silbert ${ }^{3}$ \\ ${ }^{1}$ Department of Neurology, Royal Perth Hospital, Perth, Australia \\ ${ }^{2}$ SKG Radiology, St. John of God Hospital, Perth, Australia \\ ${ }^{3}$ Perth Neurophysiology, Perth, Australia
}

The acute onset of neck pain and arm weakness is most commonly due to cervical radiculopathy or inflammatory brachial plexopathy. Rarely, extracranial vertebral artery dissection may cause radiculopathy in the absence of brainstem ischemia. We describe a case of vertebral artery dissection presenting as cervical radiculopathy in a previously healthy 43-year-old woman who presented with proximal left arm weakness and neck pain aggravated by movement. Cervical magnetic resonance imaging (MRI) and angiography revealed dissection of the left vertebral artery with an intramural hematoma compressing the left C5 and C6 nerve roots. Antiplatelet treatment was commenced, and full power returned after 2 months. Recognition of vertebral artery dissection on cervical MRI as a possible cause of cervical radiculopathy is important to avoid interventions within the intervertebral foramen such as surgery or nerve root sleeve injection. Treatment with antithrombotic agents is important to prevent secondary ischemic events.

Keywords: Vertebral artery dissection; Neck pain; Radiculopathy; Cervical spine

\section{Introduction}

In patients presenting with neck pain and arm weakness a multitude of pathologies, ranging from the benign to those potentially fatal, must be considered in the differential diagnosis. On cervical magnetic resonance imaging (MRI), the absence of discogenic or other structural causes for compression provides clinical direction to consider other causes such as inflammatory brachial plexopathy or the more recently recognised entity of vertebral artery dissection (VAD)-associated cervical radiculopathy. The typical presentation of VAD is with neck pain and occipital headache, which is often misinterpreted as musculoskeletal in nature until focal neurological signs appear [1]. Occlusion of the vertebral artery commonly results in brainstem ischemia (e.g., lateral medullary syndrome), however in a small minority of patients perfusion is unaffected and in these patients there may be evidence of cervical radiculopathy if the dissecting vertebral artery compresses the adjacent spinal nerve roots. Predisposing incidents such as sudden neck movements may be a factor in VAD, but also in discogenic cervical radiculopathies and other entities from which they must be distinguished by imaging. Identification of VAD-associated radiculopathy is essential to avoid interventions such as surgery or nerve root sleeve injection.

\section{Case Report}

A 43-year-old woman awoke with left cervical discomfort

Received Jun 10, 2012; Revised Jul 19, 2012; Accepted Sep 13, 2012

Corresponding author: Benjamin Isaac Silbert

Department of Neurology, Royal Perth Hospital, Wellington St, Perth WA 6000, Australia

Tel: +61-8-9224-2244, Fax: +61-8-9238-1321, E-mail: Benjamin.Silbert@health.wa.gov.au 
10 days before presentation. The discomfort radiated to the trapezius and scapular region, and was aggravated by neck movement. 4 days after awakening with cervical discomfort, she noted the inability to abduct her left arm. In retrospect, she recalled mild left upper cervical discomfort on neck rotation to the left while reversing her car about 4 days before the symptoms began. On examination, she had weakness involving the left deltoid, supraspinatus, infraspinatus and biceps muscles (4/5), with milder weakness of left forearm pronation and supination (4+/5). The left biceps reflex was absent and the left brachioradialis reflex reduced. The remainder of the neurological examination including left upper limb sensory examination and cranial nerve examination was normal.

Cervical MRI revealed an intramural haematoma in the left vertebral artery from mid-C7 to C2 (Figs. 1, 2). MR-angiogram (MRA) demonstrated spiral arrangement of intramural methaemoglobin and irregularity of the vessel lumen from C2-C6 (Fig. 3). No intimal flap was evident. The vessel wall haematoma was seen to compress the left C5 and C6 nerve roots. Cranial MRI was normal. An electromyogram performed 11 days after her weakness began revealed acute denervation consistent with

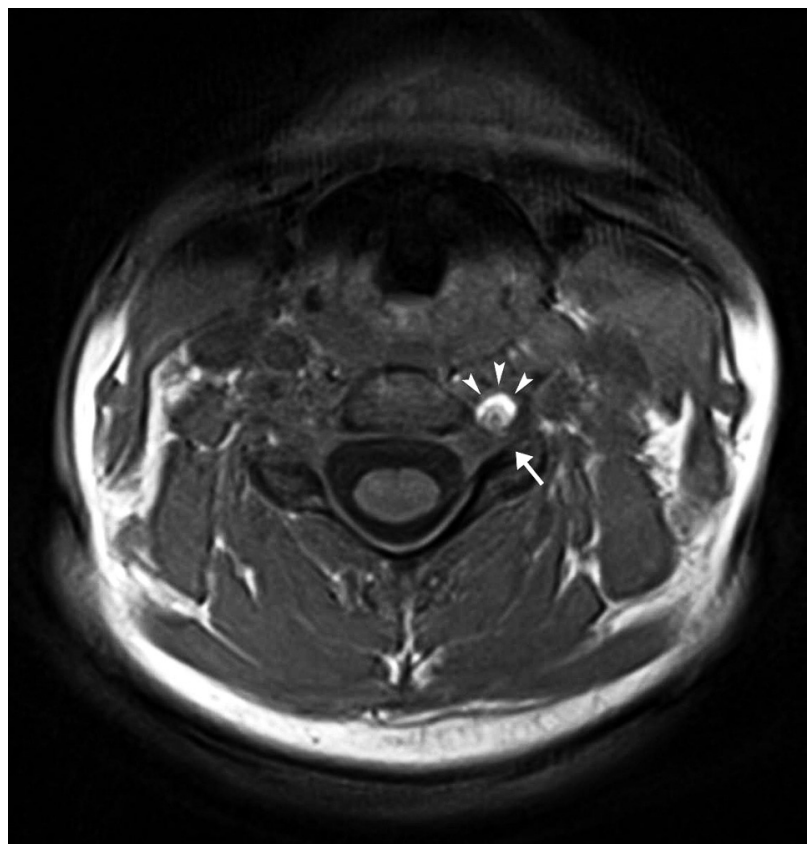

Fig. 1. Axial T1 section through the $C 4 / 5$ intervertebral foramen. The left vertebral artery (in cross-section) demonstrates marked high signal intensity anteriorly (arrowheads) with a lesser degree of high signal posteriorly. This is due to a combination of extra-cellular and intracellular methemoglobin in the vessel wall. The expanded vessel fills the foramen compressing the exiting C5 root (arrow). an acute left C5/6 radiculopathy. The lateral antebrachial sensory response and other sensory studies were normal. Nerve conduction studies including median and ulnar F-wave responses were normal. She was treated with aspirin as an antiplatelet agent. Left upper limb power returned to normal after 2 months; repeat MRI/A showed recanalization of the vertebral artery with no residual abnormality or nerve root compression.

\section{Discussion}

Acute onset of neck pain associated with arm weakness is most often due to an acute disc protrusion or uncovertebral/osteophytic disc ridge complex root compression. Less often an inflammatory brachial plexopathy may be the cause. We present an unusual case of compression of the $\mathrm{C} 5$ and $\mathrm{C} 6$ nerve roots by expansion of the vertebral artery wall within the intervertebral foramina associated with vertebral artery dissection.

Arterial dissection occurs when a tear in the intima of the vessel wall allows blood to enter the tunica media. The expanding hematoma dissects along the vessel wall; subintimal expansion stenoses the vessel lumen whereas subadventitial dissection causes aneurysmal dilatation of the vessel with the potential to compress closely related

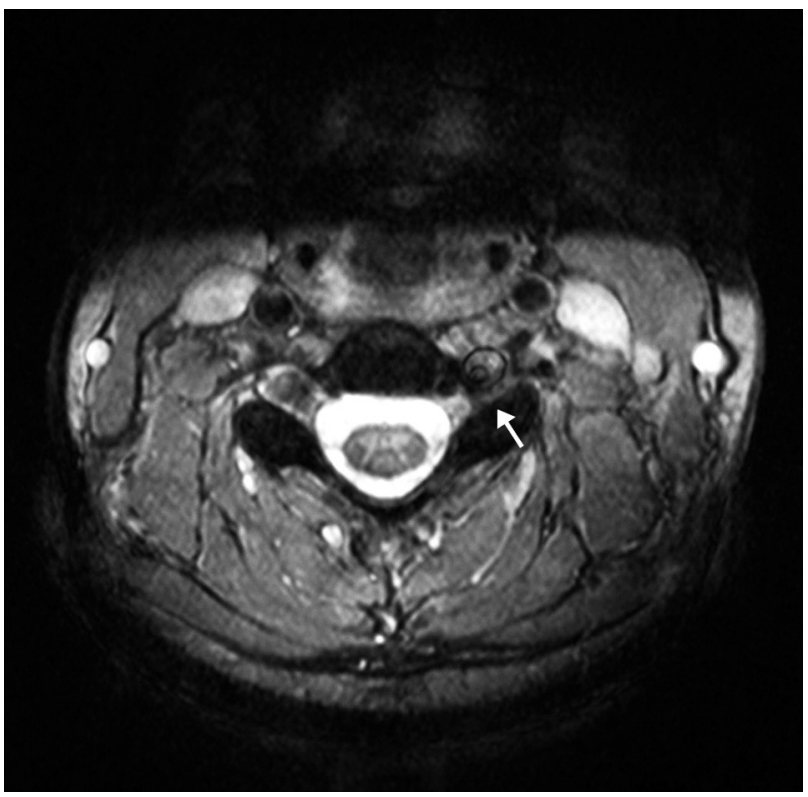

Fig. 2. Axial T2 section through nerve level in Fig. 1. The left vertebral artery lumen is narrowed (inner black circle: intima). Large crescentic high signal zone anterolaterally due to methemoglobin in the vessel wall expanding the artery (outer black circle: adventitia). The root is compressed (arrow). 




Fig. 3. Magnetic resonance angiogram study. The left vertebral artery demonstrates an irregular lumen (arrowheads) due to the spiral intramural hematoma underlying the intima. The hematoma of slightly lesser signal intensity is shown along the length of the dissected segment. The proximal and distal segments of the artery are normal. anatomical structures. The etiology of arterial dissection is not fully understood. Genetic factors such as connective tissue disorders and evidence of arteriopathy such as cystic medial degeneration and aortic root dilatation are commonly found post-mortem [1,2]. In the case of cervical arteries, when minor trauma from common activities such as coughing, sneezing or head-turning result in dissection, it is termed 'spontaneous' [1].

VAD usually presents with upper cervical pain associated with clinical evidence of brainstem or cerebellar ischemia. Presentation with acute onset neck pain and upper limb symptoms has been described less commonly [3-9]. On average, upper limb neurological symptoms develop 5 days after the onset of neck pain; the $\mathrm{C} 5$ myotome is most commonly affected and C4, C6 or C7 less often. Sensory impairment is infrequently reported. A history of minor trauma may be noted (e.g., chiropractic manipulation, forceful neck rotation or hyperextension).

The vertebral artery enters the transverse foramina of the cervical vertebrae at C6 and ascends adjacent to the ventral (motor) spinal nerve roots, supplying corresponding spinal roots via radicular arteries at each vertebral level. VAD-associated radiculopathy primarily affects the ventral spinal roots; the dorsal (sensory) spinal roots may be compressed by larger dissections or their vascular complications. Impaired perfusion of vasa nervorum and occlusion of radicular arteries by intramural hematoma (inducing neural ischemia) may also contribute [7]. The pain of VAD relates to the intramural nociceptors that receive efferent innervation from the upper cervical nerves [10]. Referred pain of VAD-associated radiculopathy at the level of C5/6 may therefore refer to the scapula as in this case, although this finding has not previously been reported.

$\mathrm{MRI} / \mathrm{A}$ is the investigation of choice, showing an enlarged vertebral artery with a spiralling crescentic rim of hyperintense signal (intramural hematoma) surrounding an eccentric hypointense signal representing the stenosed lumen [1]. The hyperintense signal corresponds to the presence of methemoglobin, and so may not be seen within the first 48 hours until sufficient metabolism of intramural blood products has occurred. Until this time, other characteristic features of dissection such as an intimal flap, false lumen, and flow-related enhancement of the residual lumen on MRA may aid diagnosis. Additionally, fat saturated axial images show an enlarged vessel wall produced by the oxy- and deoxyhemoglobin. 
Treatment with antithrombotic agents should be commenced immediately to prevent secondary ischemic events. The irregular vessel wall, false lumen and intimal flap provide a surface for thrombus formation which may then embolise or occlude the lumen. The choice between antiplatelet or anticoagulant agents is dependent on many factors including the state of the intimal lining of the vessel lumen and the presence of thrombus or embolic events [1]. For analgesia, medications other than nonsteroidal anti-inflammatories should be used to avoid potential prothrombotic interactions. The prognosis of VAD-associated radiculopathy appears favourable based on published case reports; clinical improvement and complete or partial resolution of vessel wall abnormalities is common [3-9].

VAD may present with symptoms suggestive of a cervical radiculopathy with acute onset of neck pain, a history of minor trauma and arm weakness. Diagnostic clues may be evident even on a standard cervical MRI. Recognition of VAD associated radiculopathy is essential to avoid interventions within the intervertebral foramen such as surgery or nerve root sleeve injection, and to ensure antithrombotic treatment before secondary thromboembolic events occur.

\section{Conflict of Interest}

No potential conflict of interest relevant to this article was reported.

\section{References}

1. Debette S, Leys D. Cervical-artery dissections: pre- disposing factors, diagnosis, and outcome. Lancet Neurol 2009;8:668-78.

2. Schievink WI. Spontaneous dissection of the carotid and vertebral arteries. N Engl J Med 2001;344:898906.

3. Berroir S, Sarazin M, Amarenco P. Vertebral artery dissection presenting as neuralgic amyotrophy. J Neurol Neurosurg Psychiatry 2002;72:552-3.

4. Crum B, Mokri B, Fulgham J. Spinal manifestations of vertebral artery dissection. Neurology 2000;55:304-6.

5. Dubard T, Pouchot J, Lamy C, Hier D, Caplan LR, Mas JL. Upper limb peripheral motor deficits due to extracranial vertebral artery dissection. Cerebrovasc Dis 1994;4:88-91.

6. Hardmeier M, Haller S, Steck A, Lyrer P, Engelter S, Renaud S. Vertebral artery dissection presenting with fifth cervical root (C5) radiculopathy. J Neurol 2007;254:672-3.

7. Hetzel A, Berger W, Schumacher M, Lucking $\mathrm{CH}$. Dissection of the vertebral artery with cervical nerve root lesions. J Neurol 1996;243:121-5.

8. McGillion SF, Weston-Simons S, Harvey JR. Vertebral artery dissection presenting with multilevel combined sensorimotor radiculopathy: a case report and literature review. J Spinal Disord Tech 2009;22:456-8.

9. Tabatabai G, Schober W, Ernemann U, Weller M, Kruger R. Vertebral artery dissection presenting with ispilateral acute $\mathrm{C} 5$ and $\mathrm{C} 6$ sensorimotor radiculopathy: a case report. Cases J 2008;1:139.

10. Silbert PL, Mokri B, Schievink WI. Headache and neck pain in spontaneous internal carotid and vertebral artery dissections. Neurology 1995;45:1517-22. 